Document downloaded from:

http://hdl.handle.net/10251/45030

This paper must be cited as:

García Pineda, M.; Sendra Compte, S.; Lloret, J.; Canovas Solbes, A. (2013). Saving Energy and Improving Communications using Cooperative Group-based Wireless Sensor Networks. Telecommunication Systems. 52(4):2489-2502. doi:10.1007/s11235-011-95683.

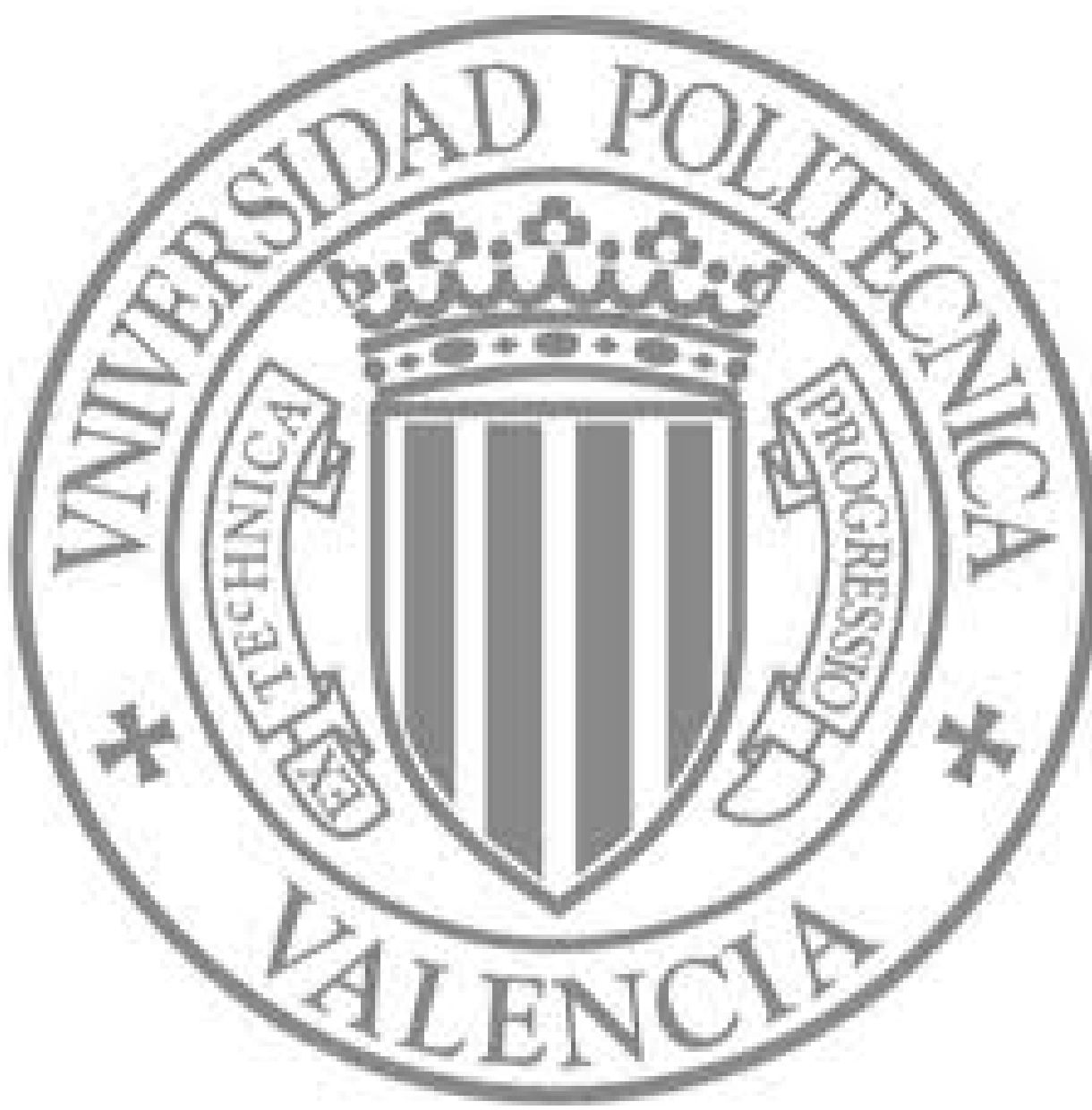

The final publication is available at

http://dx.doi.org/10.1007/s11235-011-9568-3

Copyright

Springer Verlag (Germany) 


\title{
Saving Energy and Improving Communications using Cooperative Group- based Wireless Sensor Networks
}

\author{
Miguel Garcia, Sandra Sendra, Jaime Lloret, and Alejandro Canovas \\ Research Institute for Integrated Management of Coastal Areas. \\ Universitat Politècnica de Valéncia. Camino de Vera s/n, 46015 Valencia, Spain. \\ migarpi@posgrado.upv.es, sansenco@posgrado.upv.es, jlloret@dcom.upv.es, alcasol@posgrado.upv.es
}

\begin{abstract}
Wireless Sensor Networks (WSNs) can be used in many real applications (environmental monitoring, habitat monitoring, health, etc.). The energy consumption of each sensor should be as lower as possible, and methods for grouping nodes can improve the network performance. In this work, we show how organizing sensors in cooperative groups can reduce the global energy consumption of the WSN. We will also show that a cooperative group-based network reduces the number of the messages transmitted inside the WSNs, which implieasa reduction of energy consumed by the whole network, and, consequently, an increase of the network lifetime. The simulations will show how the number of groups improves the network performance.
\end{abstract}

Keywords - Wireless Sensor Networks, Cooperative Group-based network, Saving Energy, Efficient communications.

\section{Introduction}

A WSN can be defined as a network of small embedded devices strategically located in a physical environment that are able to gather data from it. This type of structure usually consists of sensor nodes that take measurements from the environment and send the information to the base station that usually has greater processing capacity and storage [1]. The base station is considered the gateway between the sensor network and the data network. WSNs offer many good features for the users such as easy to deploy, mobility, flexibility, cost reduction and scalability [2]. The improvements in the wireless technology and the lower cost of the sensors have helped the WSN market growth.

There are many applications where the WSN could be used. Some examples are: a monitoring system for fire detection [3], for habitat monitoring [4], a fish farm monitoring and control [5], etc. 
However, when we want to cover large extensions and areas with difficult access, there are certain technical limitations that require an exhaustive network design, and study, to choose the appropriate communication algorithm and a good network topology. One of the main concerns of the researchers and developers is the whole network power consumption because devices are powered with batteries, and low maintenance is desired.

In order to reduce the power consumption, the hardware design is very important. Some of the techniques employed are related to knowing when the device must be in one mode (active mode, sleep mode or idle mode) or in another [6], thereby achieving to reduce unnecessary power consumption. But it also ensures minimum levels of quality of information flowing through the network. There are many systems, architectures, and protocols that can be used for WSN [7], but in our previous works, we have demonstrated that wireless sensors group-based topologies and networks improve the performance and the efficiency of the whole network [8][9]. Group-based topologies allow the sensor to operate more flexibly, efficiently and less energy consuming than regular network topologies [10]. Moreover, in one of our previous works we propose a group-based protocol for large wireless ad hoc and sensor networks [11].

In this paper, we demonstrate that cooperative group-based networks have very good features to be used in WSNs. Cooperative networks improve WSNs by saving energy and providing efficient communications to the WSNs.

The rest of this paper is organized as follows. Section 2 shows others architectures for saving energy in WSNs. The features of cooperative group-based networks and the differences between the regular networks are explained in Section 3. Section 4 describes our cooperative group-based architecture. A mathematical analysis to prove that cooperative group-based networks save more energy is provided in Section 5. Section 6 describes our proposal using the graph theory, and shows the number of messages needed for cooperative group-based networks. A simulation test using a network simulator is presented in Section 7. This performance test let us improve the analytical results shown in previous sections. Finally, Section 8 shows the conclusion and future work. 


\section{Related Work}

No one of the papers found in the related literature analyze the power consumption and the communications using the group-based strategy (except the in a paper from the same authors [12], where we introduce these ideas). On one hand, some works show that organizing the sensors into groups provide greater benefits than doing otherwise. On the other hand, some works are focused on network architectures to decrease the energy consumption, but without forgetting the communications efficiency.

The paper with reference [13] shows that grouping nodes in WSN gives better performance to the group and to the global system, because the system avoids unnecessary message forwarding and additional overhead. In this paper, we can see the efficiency of MANET routing protocols when the nodes are organized in groups. To do this, the authors simulate several protocols such as DSR, AODV and OLSR and study the advantages of grouping the individual nodes in each protocol for fixed and mobile networks (mobile nodes with a random behavior). Grouping nodes increase the productivity and the network performance providing low overhead and low traffic. Therefore, good scalability can be achieved in group-based networks.

J. Lloret et al. propose a new group-based protocol for WSN in [14]. First, they compare some wireless technologies (IEEE 802.11a/b/g, Bluetooth and Zigbee) for their use in WSNs. Then, they estimate the number of sensor nodes that would be needed to cover a large area and propose an analytical model comparing the energy consumed by each device over time. Finally, they compare their new group-based protocol with other protocols such as DSDV, AODV and DSR. The simulations show that the proposed protocol sends less number of packets to the network and consequently there is less energy consumption. Following these works, we see that the use of group-based networks provide benefits such as traffic, delay, etc. in networks.

There are also several published works related with the analysis of energy consumption and energy saving mechanisms in WLANs. These types of analysis may help us know some power saving issues when they are used in WSNs.

The paper in reference [15] describes several techniques to reduce the dynamic energy consumption in WLAN IEEE 802.11n standard systems in order to increase the battery life in mobile systems. They propose to reduce the devices 
consumption from their initial design by building smaller devices because they consume less energy.

V. Raghunathan et al. describe in [16] some considerations about the architecture and protocols to be considered in order to make an energy-efficient design of a sensor node in a WSN. The paper shows an analysis of the energy consumption characteristics of a node, using multihop architectures. Moreover, they identify the key factors that can affect the life of the global system. They show the sensor energy consumption, the power-aware computing, the energy-aware software, the power management of wireless communication, the energy-aware packet forwarding and traffic distribution, among others. This work is important to know what the most important factors are, in order to take them into account when a system is developed.

In paper [17], the authors discuss the required key technologies for low-energy distributed microsensors and present a power aware Application Programming Interface (API) to calibrate the energy efficiency of various parts of the application. They took care of the power aware computation/communication component technology, low-energy signaling and networking, system partitioning considering computation and communication trade-offs, and a power aware software infrastructure. This work also shows some analytical models of the device behavior and the results of applying different parameters, such as the number of sensors, type of protocol used to communicate the sensors, the system power consumption by controlling when they should enter a sleep mode and applying dynamic voltage scaling. These models will help us to develop our models.

Reference [18] shows the relationship between the power usage and the number of neighbors in a WSN. They state that many of the topologies proposed for wired networks cannot be used for wireless networks because wireless networks depend on the physical neighborhood and the transmission power while in a wired network depends on the physical connections among nodes. A. Salhieh et al. analyze various $2 \mathrm{D}$ and $3 \mathrm{D}$ structures with different numbers of nodes. They measured the network performance for different network topologies and the node's power dissipation in order to determine what the best topology is for a WSN. But, they assume that they control the placement of these sensors and the sensor locations are fixed respect to each other. Moreover, the authors do not 
consider the effects of communication with a base station. They conclude that the best power efficiency in $2 \mathrm{D}$ topologies is achieved with four neighbors, and although 3D topology is better, it may not be feasible for some applications.

The energy consumption must not deteriorate the communications quality. We have found some works that attempt to improve the efficiency of the communications in WSNs, but trying to reduce energy consumption. Many of them are related with the development of protocols at the MAC layer. Here, we present some works.

In [19], S. Jayashree et al. present a MAC protocol that takes into account the state of the battery and improves its efficiency. The main objectives of this protocol are to reduce energy consumption, to extend the battery life and to have higher performance. They present the BAMAC model battery that is based on Markov chain process. Each node contains a table with the information of the battery charge of each neighbor that is from a jump. The information in the table organizes battery charges in descending order. RTS, CTS, Data and ACK packets carry information of the remaining battery charge of the node that originated the packet. A node that listens one of these packets, collects and stores the information in its table. The simulation results, of this work, show that the battery life is prolonged in about $70 \%$ and reduces the nominal battery consumption for packet transmission by $21 \%$ compared with IEEE 802.11 MAC protocols DWOP. In [20], the authors present sensor-MAC (S-MAC), a new MAC protocol designed for WSN. First, the authors identify the main causes of energy consumption: (1) when a transmitted packet is corrupted and it has to be discarded because retransmissions increase the energy consumption, (2) the overhearing (a node picks up packets that are destined to other nodes), (3) the control packets due to the repetitive sending and receiving, and (4) being listening because measurements have shown that this functional mode consumes around 50-100\% of the energy required for receiving. The main goal of this MAC protocol is to reduce the energy consumption by taking care of these main causes, while supporting good scalability and collision avoidance. The authors show that their protocol achieves a reduction of the $30 \%$ in the device consumption and the protocol has the ability to make trade-offs between energy.

C. Ching and C. Schindelhauer, presented in [21] a proposal that takes into account the power consumption of both the motion and the radio communications 
because they are the primary energy consumers. In order to achieve their goal, they used a scenario with a series of robots and several antennas that communicate among themselves. Each robot is assigned to different tasks (searching, exploring, sensing, foraging, etc.). The authors reduce the energy consumption in mobile receivers within a WSN considering a hybrid wireless network formed by two scenarios: a single autonomous mobile node communicating with multiple static relays through single hop, and secondly, a single mobile node communicating with a static base station via a mobile relay. The mobile node interacts with the relays within its vicinity by continuously transmitting high-bandwidth data. The authors introduce Radio-Energy-Aware (REA) path computation strategy and propose a novel energy-aware that finds the best paths. The energy consumption for both mobility and communications is minimized for mobile receivers and for the devices that keep communications in all mobile nodes. They compare their method REA with Motion-Energy-Aware (MEA) method. The simulation results show that their proposed strategy improves the energy efficiency of mobile nodes.

The paper with reference [22], authored by Q. Gao et al., show the relationship between optimal radio range and traffic. The authors adjust the communication systems properties, according to some optimum strategies, in order to save the average power. They carry out different approaches and mathematical developments in order to adjust the maximum performance of a sensor node model. The formulae used are made taking into account factors such as: the network size, and the distance between nodes and between the nodes and the base station. The authors proved that a good design and node's positioning choice inside the WSN could lead to double the network lifetime because of the significant reduction of the energy consumption.

In [23], the authors analyze the broadcast routing according to the energy cost. In this case, the energy cost is defined as the sum of each individual node energy cost that transmits broadcast messages. So, in order to solve this problem, the authors try to minimize the number of broadcast messages. They use three centralized heuristic methods to minimize it. According to their simulations, we can see that there are some algorithms better than others. But the main problem of these solutions is that all of them are based on centralized solutions and nowadays the architectures are evolving to decentralized solutions. 
Tiago Camilo et al. present in [24] a new routing protocol specially designed to maximize the life time of the sensor nodes in WSNs. This protocol is based on Ant Colony Optimization (ACO) metaheuristic method. In this protocol, the communication with the next network node is selected according to a probability (which is function of the node energy and the number of nodes of the path). In order to check the truthfulness of their proposal, they present some simulations that compare their protocol with other ant-routing protocols. The main drawback of this type of protocols is the management of the ants (the backbone of routing protocol).

In [25], the authors show that the cluster-based sensor networks have a better energetic behavior than regular sensor networks. They present a routing protocol for managing the sensor network with the main objective of extending the life of the sensors in a particular cluster. Their proposal uses a gateway node which acts as a cluster-based centralized network manager that sets routes for sensor data, monitors latency throughout the cluster, and arbitrates medium access among sensors. Finally they present some simulations that show the benefits of their algorithm.

Another type of sensor lifetime extension mechanism is the one based on deployment strategies. In [26], the authors explore these strategies. They propose a general framework for the analysis of the network lifetime for several network deployment strategies, and the authors consider the extra costs associated with each deployment strategy to determine the best overall strategy for a given scenario. This is an important work because it shows several issues that influence the node's lifetime, and demonstrates and disagrees some intuitions.

Finally, another work where the authors present an energy-efficient deployment algorithm based on Voronoi diagrams is [27]. Three different deployment methods are proposed by the authors. The first one is a deployment algorithm for mobile nodes where each node is equally important and a peer-based structure is obtained. The second one uses the clustering idea to increase the amount of local control. The third one uses the algorithm based on Voronoi diagrams to provide an estimation of the lifetime of each node in a distributed fashion. They simulate their algorithm and check its benefits, but the main problem of this research is that it is only based on one-hope architecture and nowadays almost all networks are multi-hop networks. 
None of the previous works have used cooperative group-based sensor network strategies in order to save energy or improve the communications in the WSN. For this reason, we presented a main idea in [12]. In this paper, we show the analytical model and its simulations which describe the improvement of cooperative groupbased systems versus regular architectures.

\section{Differences between Regular WSNs, Group-based WSNs and Cooperative Group-based WSNs}

In this section we review the main differences between regular WSNs and cooperative group-based WSNs.

Regular WSNs are networks where all nodes have the same function from the point of view of the network level. Each node measures a parameter, then, it is processed and, finally, the information is sent to its neighbors. Its neighbors will send the sensed information to their neighbors in order to reach the base station or the sink.

Passive WSNs are those ones whose sensors sense the environment and send the information to a sink without taking any other action. In an active WSN, when an event occurs, it is notified to the sink and/or to the manage center (MC). Then, the MC sends the necessary information to all nodes (or to some selected nodes) of the network in order to take the appropriate action (send the information to other nodes, sense more variables, etc.).

Currently passive WSN are not useful because in many environments an intelligent WSN is required. Sometimes, when an event occurs inside the WSN certain tasks should be carried out to perform a specific reply. For example, if we build a WSN to detect fire, the sensors should be able to sense different variables in order to verify the fire and even to monitor it (temperature, $\mathrm{CO}_{2}$, humidity, wind direction, etc.). In this case, sensors may collect these variables and, after some data processing, send the necessary information to a higher processing capacity node in order to activate the appropriate fire fighting mechanisms.

Figure 1 shows the differences between passive and active WSNs. Red arrows indicate the messages from sensor nodes to the MC and the blue arrows indicate the communication from the $\mathrm{MC}$ to the sensor nodes in order to take the appropriate actions. Blue arrows only exist in active WSNs. 

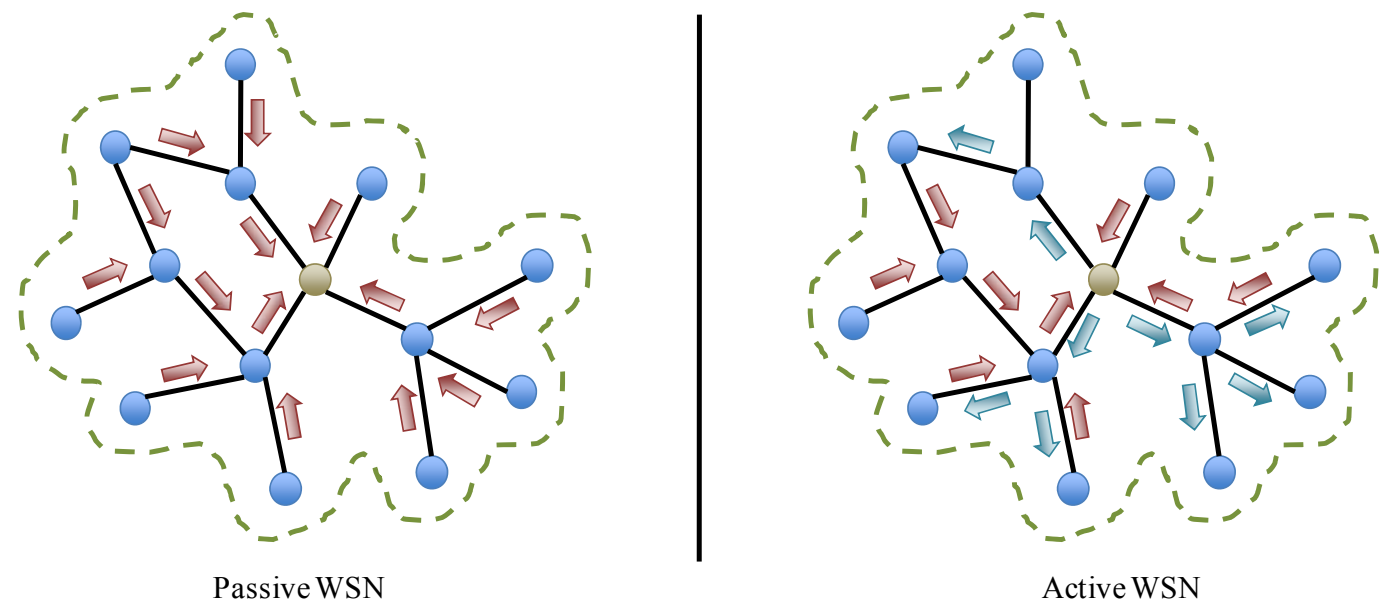

Figure 1. Passive WSN vs. Active WSN

Cooperative group-based sensor networks do not work in the same way. First, they are group-based networks, so the network is logically divided into several small networks (groups). A group is defined as a small number of interdependent nodes with complementary operations that interact in order to share resources or computation time, or to acquire content or data and produce joint results. In a wireless group-based architecture, a group consists of a set of nodes that are close to each other (in terms of geographical location, coverage area or round trip time) and neighboring groups could be connected if a node of a group is close to a node of another group. In [12], we can see that the group-based WSNs provide many benefits. Moreover, cluster-based networks could be considered as a subset of the group-based networks, because every cluster could be a group [28][11]. But, a group-based network is capable of having any type of topology inside the group, not only clusters. Furthermore, in a group-based network, each group could use a different type of routing protocol. A cluster-based network is made of a $\mathrm{CH}$, Gateways, and Cluster Members (CM). In a cluster, $\mathrm{CH}$ nodes fully control the cluster, while in group-based networks, no node controls the group.

Cooperative group-based sensor networks imply cooperation between nodes from the same group and cooperation between nodes from different groups. Thus, the information may be shared only between the most appropriate nodes (from the same groups or from different groups). An event produced by a sensor will imply the exchange of information between different nodes in order to take a final decision and produce an action in the same place or in other location of the network. Moreover, in a cooperative WSN only those parameters that can affect 
the final decision, i.e. the information coming from the neighboring groups of the group that generated the alert, are considered. Following the example of the WSN deployment for fire detection, when a sensor detects a fire, the alert message is sent to all nodes in its group, and, after processing the information, and taking into account other parameters such as wind direction, the alert is sent to the affected neighboring groups. Affected neighboring groups will perform the appropriate actions that other groups will not have to do.

From the point of view of the network messages and energy consumed, in a regular WSN, when a node registers an alert, it transmits the alert to all its neighbors, these neighbors transmits the alert to their neighbors and so on, the alert is spread to the entire WSN without control. Moreover, a node could receive several times the alert message from different neighbors (see Figure 2a). This situation leads to excessive energy consumption. A collaborative group-based WSN is built based on defined areas or as a function of the nodes' features. Moreover, each group is formed by nodes that interact to share resources or to acquire data to produce joint results [29]. In this case, when a sensor detects a new event, this sensor sends the information to all the members of the group and, depending on the case, the neighboring groups could share this information in order to reach all sensors of the WSN or just some groups. Only the closest sensors to the edge of the group will transmit the information to the sensors of other groups (see Figure $2 \mathrm{~b}$ ). This fact avoids raising considerably the global energy consumption of the WSN, which is very important to enlarge the lifetime of the WSN.

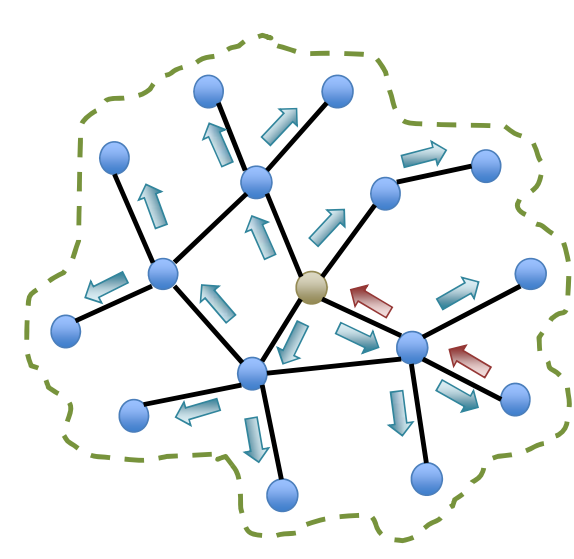

a)

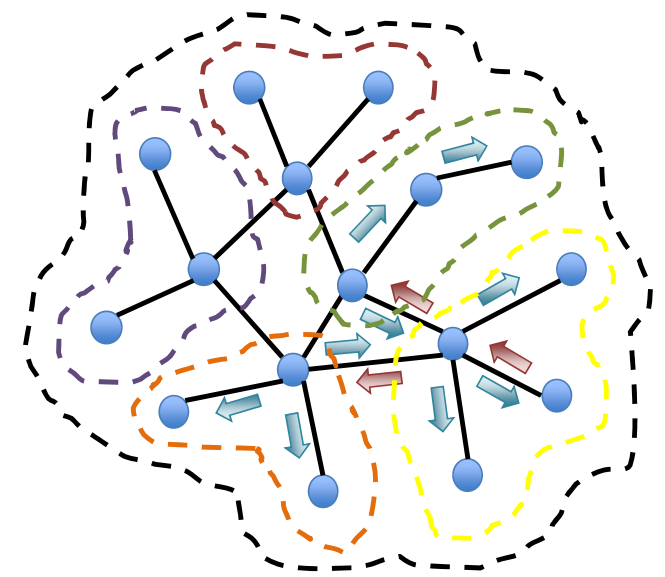

b)

Figure 2. Regular WSN vs. Cooperative group-based WSN. 
Although we are mainly talking about collaborative group-based WSN with fixed sensors, they could be mobile sensors. In our study we will consider group-based WSNs where mobile sensors move only inside the boundaries of the groups. Failures could happen in each group. In this case, the mobility will affect in the same manner as in a non-group-based WSN.

In [11] we presented an example of cooperative group-based sensor network. The WSN formation is performed in the same manner of a group-based network but introducing cooperation issues. In addition, each group selects the best connection between sensor nodes taking into account the proximity and the nodes' capacity [30]. In order to have an efficient group-based wireless sensor network, the groups have to communicate with their neighboring groups. When a node detects an event, it warns the alert, jointly with the parameters measured, to the nodes of its groups and, routing the information, to its neighboring groups (not to all groups) based on the location of each group or any other parameter. The location of the sensors could be entered manually or using GPS [31], and a position-based routing algorithm [32] could be used to send the message to the appropriate situation. Neighboring groups could reply to the group that firstly sent the alert if any of the parameters that caused the alert is changed, in order to take the appropriate actions. Cooperation with other groups could change the direction of the alert propagation and the level of the alert.

Figure 3 a) shows a group-based topology example. In a group-based WSN, all groups will be aware of an event produced inside the Group 1. The network efficiency would be yet higher than in a regular topology [10]. But, in cooperative group-based networks this efficiency is greater, because the decision is taken based on the information shared and only the neighboring groups will be aware of it. The other groups could be in sleep mode. Sleep groups will be saving energy and they would not transmit unnecessary information. Figure $3 \mathrm{~b}$ ) shows that the neighboring cooperative groups of group 1 are group 2 and 5. In this case they are the physical neighbors. An example is explained in more detailed in [29]. 


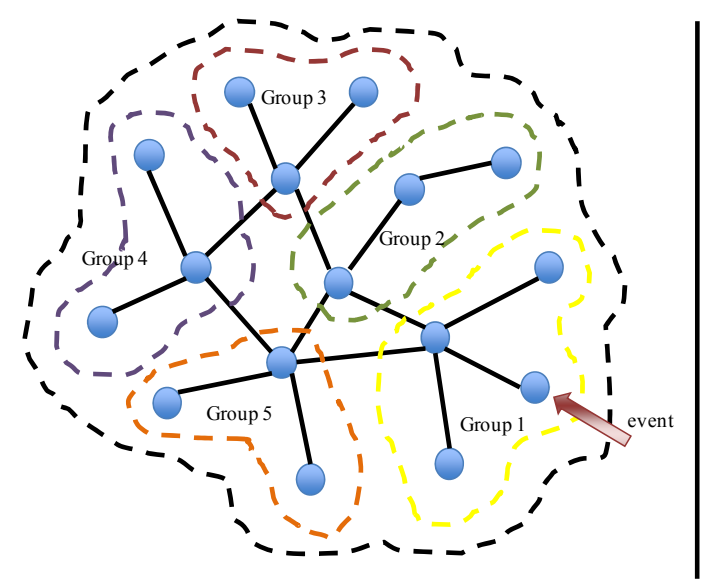

a) Group-based WSN

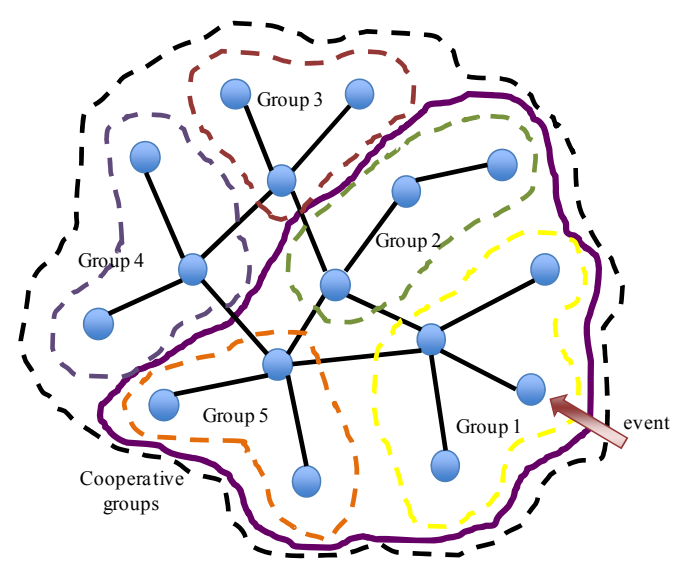

b) Cooperative Group-based WSN

Figure 3. Comparison of group-based WSN and cooperative group-based WSN.

\section{Energy Analysis}

This section analyzes the energy needed to transmit packets in a cooperative group-based WSNs architecture and we compare it with a regular WSN architecture.

The notation used in our analysis is shown in table 1. $\varphi_{11}, \varphi_{12}$ and $\varphi_{2}$ are constant radio parameters, typical values are $\varphi_{11}=50 \mathrm{~nJ} / \mathrm{bit}, \varphi_{12}=50 \mathrm{~nJ} / \mathrm{bit}, \varphi_{2}=10$ $\mathrm{pJ} / \mathrm{bit} / \mathrm{m}^{2}$ (when $\mathrm{n}=2$ ) or $0.0013 \mathrm{pJ} / \mathrm{bit} / \mathrm{m}^{4}$ (when $\left.\mathrm{n}=4\right)$.

Table 1. Notation and definition.

\begin{tabular}{|c|c|c|c|}
\hline Parameter & Definition & Parameter & Definition \\
\hline$\varphi_{11}$ & Power required to turn on the transmitter & $\mathrm{r}$ & Bit rate (bits per second) \\
\hline$\varphi_{12}$ & Power required to turn on the receiver & $\mathrm{R}$ & Average area radius of a WSN \\
\hline$\varphi_{1}$ & $\varphi_{11}+\varphi_{12}$ & $\mathrm{R}_{\mathrm{i}}$ & $\begin{array}{l}\text { Average area radius of the } \\
\text { group }_{i}\end{array}$ \\
\hline$\varphi_{2}$ & Power required to transmit & $\mathrm{S}$ & \# of sensors in the WSN \\
\hline $\mathrm{d}$ & $\begin{array}{l}\text { Distance between } 2 \text { communicating } \\
\text { sensors }\end{array}$ & $\mathrm{S}_{\mathrm{i}}$ & $\#$ of sensors in the group ${ }_{i}$ \\
\hline $\mathrm{d}_{\mathrm{opt}}$ & Optimum distance between 2 sensors & $\mathrm{J}_{\mathrm{m}}$ & \# of cooperative groups \\
\hline $\mathrm{n}$ & Path loss exponent (typical values: 2 or 4 ) & $\mathrm{J}$ & $\#$ of groups, $\mathrm{m} \in[1, \mathrm{~J}]$ \\
\hline
\end{tabular}

We follow the model presented in [33]. The energy consumed by a sensor to transmit and receive a data packet between two nodes is given by (1).

$$
P=\left(\varphi_{11}+\varphi_{12}\right) \cdot r+\varphi_{2} \cdot\left(d^{n}\right) \cdot r
$$

Where $d$ is the distance between both nodes. 
Let be $\mathrm{D}$ the distance between the sending node of a group and the closest node from other group. Thus, $P(D) \geq P^{\text {opt }}(D)$, being $P^{\text {opt }}(D)$ the minimum power to transmit a data packet from the node to the other group. $P^{o p t}(D)$ is equal to (2) if and only if D is multiple integer of $d_{o p t}=\sqrt[n]{\frac{\varphi_{1}}{\varphi_{2} \cdot(n-1)}}$, as we can see in [34].

$$
P^{o p t}(D)=\left(\varphi_{1} \cdot \frac{n}{n-1} \cdot \frac{D}{d_{o p t}}-\varphi_{12}\right) \cdot r
$$

$P^{o p t}(D)$ is the lower bound of energy consumption in the flat scheme without data aggregation, which indicates an ideal case where the per-hop distance for transmission is $d_{\text {opt }}$ meters. According to the energy model in [33] and the previous assumptions, the expected energy consumption per second of a group is given by (3).

$$
P=\left(S_{i}-1\right) \cdot\left(\varphi_{1}+\varphi_{2} \cdot \frac{2 \cdot R_{i}^{n}}{n+2}\right) \cdot r+\left(\varphi_{11}+\varphi_{2} \cdot D^{n}\right) \cdot r
$$

We assume that there are $J$ groups in the network and each regular node only needs to transmit its data packet to the central node or to the border node of its group. The average radius of each $m$ group can regard it as $R / \sqrt{J}_{m}$, and the expected energy consumption per second in all regular nodes will be given by (4).

$$
P_{r n}=\left(s-J_{m}\right)\left[\varphi_{11}+\varphi_{2} \cdot \frac{2}{n+2} \cdot\left(\frac{R}{\sqrt{J}}\right)^{n}\right] \cdot r
$$

The energy consumption for all border nodes is given by (5).

$$
P_{b o r}=\left(s-J_{m}\right) \cdot \varphi_{12} \cdot r+J_{m} \cdot\left[\varphi_{11}+\varphi_{2} \cdot\left(\frac{R}{\sqrt{J}}\right)^{n}\right] \cdot r
$$

In order to evaluate the energy consumed in a network without cooperative groups, we consider (4) and (5) for a single group $(J=1)$, and only one sink node. Then, we obtain the equations (6) and (7) respectively.

$$
\begin{gathered}
P_{r n}=s \cdot\left[\varphi_{11}+\varphi_{2} \cdot \frac{2}{n+2} \cdot(R)^{n}\right] \cdot r \\
P_{b o r}=\left[s \cdot \varphi_{12}+\varphi_{11}+\varphi_{2} \cdot R^{n}\right] \cdot r
\end{gathered}
$$


The global energy consumption in both cases cooperative network and a network without cooperative groups is given by equation (8).

$$
P_{T}=P_{r n}+P_{b o r}
$$

Another important issue that we have noticed is the importance of the number of group nodes (high $J$ ) than the number of cooperative groups $\left(J_{m}\right)$. If the number of cooperative groups is greater, the consumption will be lower, but this consumption decreases more quickly if we create more groups.

\section{Analytical comparison}

In order to observe the energy-saving improvements provided by a cooperative group-based WSN compared to regular WSN, we have taken into account equation 8 for both cases (regular WSN and cooperative group-based WSN). Following the typical values of the constants that appear at the beginning of this section $\left(\varphi_{11}=50 \mathrm{~nJ} / \mathrm{bit}, \varphi_{12}=50 \mathrm{~nJ} / \mathrm{bit}, \varphi_{2}=10 \mathrm{pJ} / \mathrm{bit} / \mathrm{m}^{2}\right)$, we have used $\mathrm{n}=2$ because it is the most appropriate value for outdoor communications. Furthermore, we have chosen $\mathrm{m}=2$, our network has 100 nodes $(\mathrm{s}=100)$ and the network radius equals to 200 meters $(\mathrm{R}=200)$. We have programed the above equations and varying analytically the number of groups and bitrate we obtain the Figure 4. Figure 4 shows the global energy consumption for different values of $J_{m}$. When $J_{m}=1$, we talk about just one group (the whole WSN), so we can see that the energy consumption is higher than in any other value of $J_{m}$ (higher values of $J_{m}$ imply more groups). By increasing $J_{m}$ we see that consumption decreases, but each time the difference of the slope is lower.

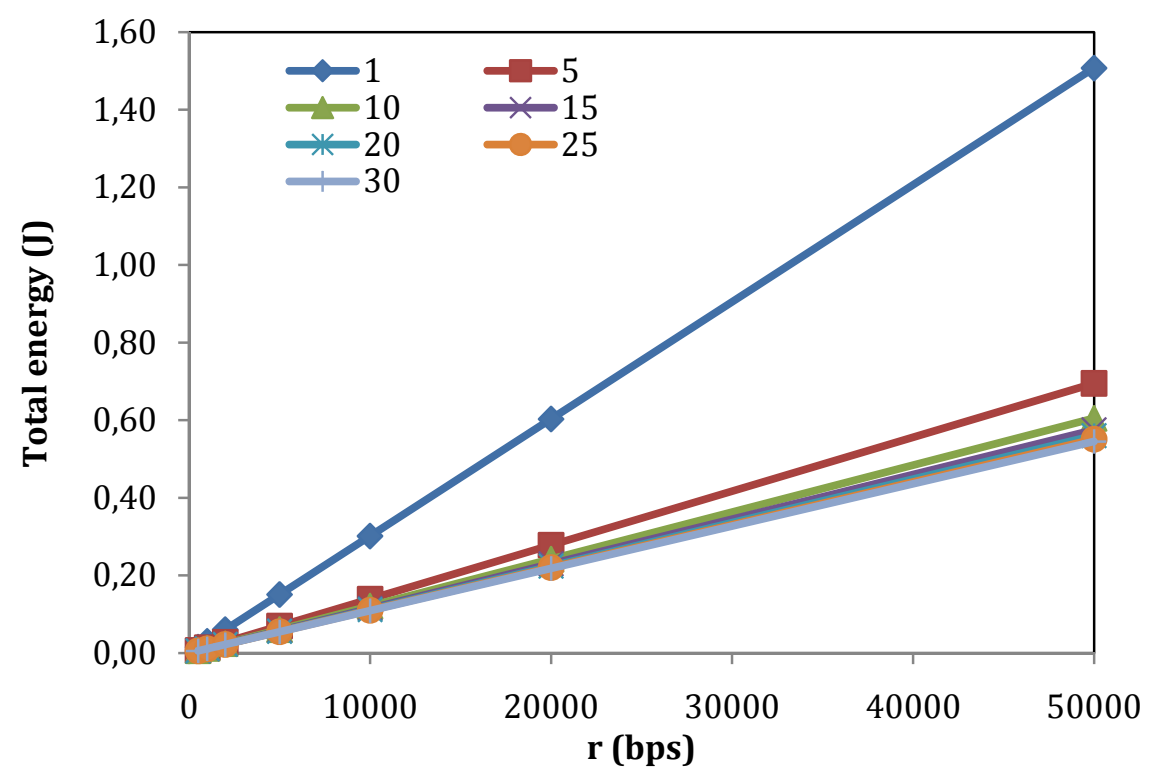


Figure 4. Energy of the cooperative group-based WSN.

\section{Communication Analysis}

In a cooperative group-based WSN all nodes are alike from of the point of view of network level and may be mobile. There are not base stations, sinks or management nodes. For this reason, all nodes can make decisions of their connections according to an algorithm. In our case the algorithm is defined in [29]. Moreover, all communications between the sensor nodes are done by wireless links.

As usual, we can model a WSN using the graph theory, where a WSN is defined as $G=(S, E)$, where $\mathrm{S}$ is the set of sensor nodes $(|S|=s)$ and $e=(i, j) \in E$ represents a wireless link between the nodes $i$ and $j$ only if they are in their communication range.

Any two nodes that are connected by an edge are neighbors to each other. Nodes in $G$ can send (and receive) messages to (from) their neighbors. Every node $v \in S$ has a unique identity $I D(v)$. Each node initially knows its own identity and the identities of its neighbors in one jump inside $G$.

The size of a group is the number of nodes belonging to it. According to the definition of our WSN, there are three types of nodes: central, intermediate and border nodes [10]. For example, the central node of the group $k$ is defined as $C(k)$. The number of border nodes in the group is $B$, where $B$ is smaller than $n$.

Other parameters that are used for defining communications in sensor networks is the distance between two nodes, $d(i, j)$, it is the Euclidean distance between the nodes $i$ and $j$. As it is a group-based network, each group will be defined as $G(k)$, in this case this is the group $k$.

From the graph theory textbook [35] we will denote $\Gamma_{k}(i)$ the k-neighborhood of a node $i$, e.g., $\Gamma_{k}(i)=\{v \in S \mid 0<d(i, j) \leq k\}$ and we will denote $\delta_{k}(i)=\left|\Gamma_{k}(i)\right|$. Besides, we will denote $e(i / G)=\max _{v \in G(i)}(d(i, j))$ the eccentricity of a node $i$ inside its group. Thus the diameter of a group will be $D(G(i))=\max _{v \in G(i)}(e(v / G))$. Once the definition of a group-based WSN has been done, we analyze the number of messages sent inside the network when an event occurs and this information has to be sent to other nodes. According to [36], in the worst situation, the number 
of messages in a regular network when there is an alert is $O(s)$, while in a cooperative group-based network the number of messages is $O\left((m+1) B^{2}\right)$, where $m$ is the average number of cooperative groups in the network.

If $B<<s$ we can affirm that the number of messages in a group-based network will be less than in regular networks, for this reason $O\left((m+1) B^{2}\right)<<O(s)$.

\section{Analytical comparison}

In order to validate this analysis, we performed a simulation test. There were $s$ nodes distributed in a square area of length $l$ units. The nodes are randomly placed. The average density of nodes per unit length is $s / l^{2}$. In this model, the nodes have a link with another node if and only if they are within a distance $d$ units of each other. Consequently, a node will have an average of $\left(\pi d^{2} s / l^{2}\right)-1$ neighbors. This leads to an edge probability of $\left(\pi d^{2} s / l^{2}-1\right) /(s-1)$ which is the probability that two nodes chosen at random in the network are connected by a link. In particular, for a large value of $s$, the edge probability is approximately equal to $\pi d^{2} / l^{2}$.

Table 2 shows the average number of border nodes, the average number of groups, the likelihood of border nodes, the number of messages that will be in the case of $l=25$ units of length and $d=1$ unit and the percentage of improvement respect to a regular architecture (without groups). The observed data in this table have been obtained using the previous boot parameters and applying the formulas presented in this section. We have used a mathematical program where the formulas have been programed and then we have introduced the different starting values for obtaining these results (see Table 2). We have estimated all these parameters when $B$ has 1, 2, 4, 8 and 16 nodes, with an average number of cooperative groups $(\mathrm{m})$ ranging from 0 to 5 (we have selected $0,1,3$ and 5), and when the number of nodes in the whole WSN has 100, 200 and 500.

Table 2. Results of the communication analysis.

\begin{tabular}{|c|c|c|c|c|c|c|}
\hline & $\mathbf{s}$ & $\begin{array}{c}\text { Average } \\
\text { number of } \\
\text { border nodes }\end{array}$ & $\begin{array}{c}\text { Average } \\
\text { number of } \\
\text { groups }\end{array}$ & $\begin{array}{c}\text { Likelihood } \\
\text { of border } \\
\text { nodes }\end{array}$ & $\begin{array}{c}\text { Number of } \\
\text { messages }\end{array}$ & $\begin{array}{c}\text { Percentage } \\
\text { of } \\
\text { improvement }\end{array}$ \\
\hline $\mathbf{B}=\mathbf{1 ,}$ & $\mathbf{2 5 0}$ & 0.256 & - & 0.0001028 & 250 & - \\
\hline
\end{tabular}




\begin{tabular}{|c|c|c|c|c|c|c|}
\hline \multirow[t]{2}{*}{$\mathbf{m}=\mathbf{0}$} & 500 & 1.512 & - & 0.00303 & 500 & - \\
\hline & 1000 & 4.024 & - & 0.00403 & 1000 & - \\
\hline \multirow{3}{*}{$\begin{array}{l}B=2, \\
\mathbf{m}=1\end{array}$} & 250 & 0.256 & 125 & 0.0001028 & 8 & $96.8 \%$ \\
\hline & 500 & 1.512 & 250 & 0.00303 & 8 & $98.4 \%$ \\
\hline & 1000 & 4.024 & 500 & 0.00403 & 8 & $99.2 \%$ \\
\hline \multirow{3}{*}{$\begin{array}{l}B=4, \\
\mathbf{m}=3\end{array}$} & 250 & 0.256 & 62.5 & 0.0001028 & 64 & $74.4 \%$ \\
\hline & 500 & 1.512 & 125 & 0.00303 & 64 & $87.2 \%$ \\
\hline & 1000 & 4.024 & 250 & 0.00403 & 64 & $93.6 \%$ \\
\hline \multirow{3}{*}{$\begin{array}{l}B=8, \\
m=3\end{array}$} & 250 & 0.256 & 31.25 & 0.0001028 & 256 & $\begin{array}{c}\text { No } \\
\text { improvement }\end{array}$ \\
\hline & 500 & 1.512 & 62.5 & 0.00303 & 256 & $48.8 \%$ \\
\hline & 1000 & 4.024 & 125 & 0.00403 & 256 & $74.4 \%$ \\
\hline \multirow{3}{*}{$\begin{array}{c}B=16, \\
m=5\end{array}$} & 250 & 0.256 & 15.625 & 0.0001028 & 1536 & $\begin{array}{c}\text { No } \\
\text { Improvement }\end{array}$ \\
\hline & 500 & 1.512 & 31.25 & 0.00303 & 1536 & $\begin{array}{c}\text { No } \\
\text { improvement }\end{array}$ \\
\hline & 1000 & 4.024 & 62.5 & 0.00403 & 1536 & $\begin{array}{c}\text { No } \\
\text { improvement }\end{array}$ \\
\hline
\end{tabular}

According to the results shown in Table 2, when we have a cooperative groupbased WSN with $\mathrm{B}=4$ and $\mathrm{m}=3$ or $\mathrm{B}=8$ and $\mathrm{m}=3$, the average number of nodes is adequate, but when there are many border nodes the efficiency of the collaborative group-based WSN is low (in red in table 2, e.g. $\mathrm{B}=16$ and $\mathrm{m}=5$ ). This happens because when the groups are large, $B \uparrow \uparrow$, so the WSN becomes a regular WSN and moreover the architecture needs more control messages to manage the collaborative group-based architecture. There is a tradeoff between the number of network nodes and the appropriate number of groups, so a groupbased network is better than the regular network.

Figure 5 shows the maximum number of broadcasting messages needed when we have several sizes of groups and several average cooperative groups. In this case the number of nodes $s$ was 1000 and $l$ was 25 units. In this figure we can observe that increasing the group size $(B)$ and the number of cooperative groups, it increases the number of messages in the WSN. The simulation shows that when a high number of messages is expected in the WSN, it is better a group-based WSN with a low $B$ and with few cooperative groups. This happens with the broadcasting messages, but if we look the management messages, when we have small groups there are a lot control messages to create and manage the groups. For 
this reason, there has to be a compromise between the group size, the number of cooperative groups and the number of broadcasting messages. Seeing the figure 5, the best solution could be a group size between 4 and 6 and an average number of cooperative groups between 3 and 5 .

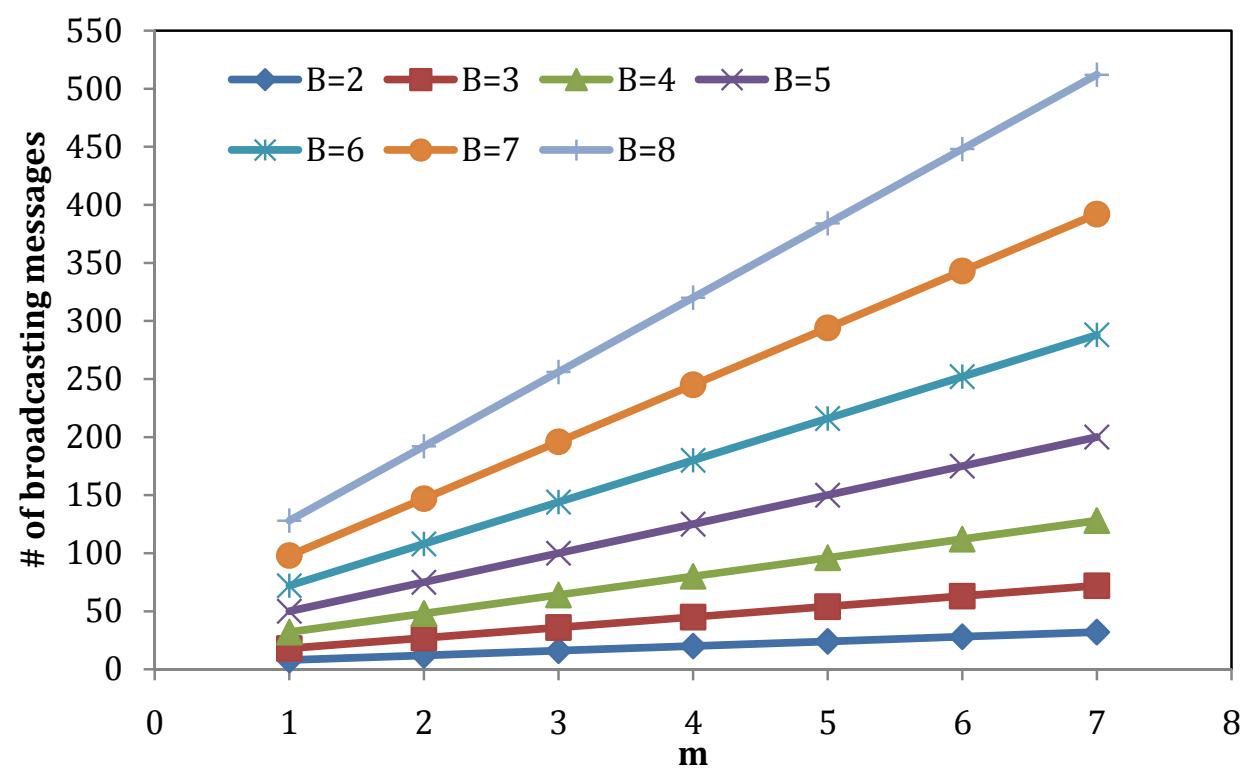

Figure 5. Maximum number of broadcasting messages for different group size and varying the average number of cooperative groups.

\section{Cooperative group-based network test with mobility}

In order to evaluate the system proposed in this paper. We have simulated several scenarios using the OPNET Modeler network simulator [37]. In next simulations we are going to see the behavior of the cooperative group-based WSN according to the number of groups in network. The test scenario has 100 sensor nodes placed in 500x500 meters. We have increased the number of groups in each simulation. We have chosen DSR as routing protocol because in [14] we saw that this protocol has the worst behavior. We select the worst routing protocol because we want to show the positive aspects of our system, even using poor conditions.

Instead of a standard structure we have chosen a random topology. The nodes can move randomly during the simulation. The physical topology does not follow any known pattern. The obtained data neither depend on the initial topology of the nodes nor on their movement pattern because all of it has been fortuitous.

The sensor nodes have a $40 \mathrm{MHz}$ processor, 512KB RAM memory, a radio channel bandwidth of $1 \mathrm{Mbps}$ and their working frequency is $2.4 \mathrm{GHz}$. Their maximum coverage radius is 50 meters. This is a conservative value because 
usually the nodes in sensor networks have larger coverage radius, but we preferred to have lower transmitting power for the sensor devices in order to enlarge their lifetime.

The traffic load used in the simulations is MANET traffic generated by OPNET. We inject this traffic 100 seconds after the beginning. The traffic follows a Poisson distribution (for the arrivals) with a mean time between arrivals of 30 seconds. The packet size follows an exponential distribution with a mean value of 1024 bits. The injected traffic has a random destination address, obtaining a simulation that does not depend on the traffic direction. In Figure 6 we see that the traffic injected into the simulation follows the same pattern for all scenarios. The average traffic is $100 \mathrm{Kbps}$ (an adequate traffic for WSNs).

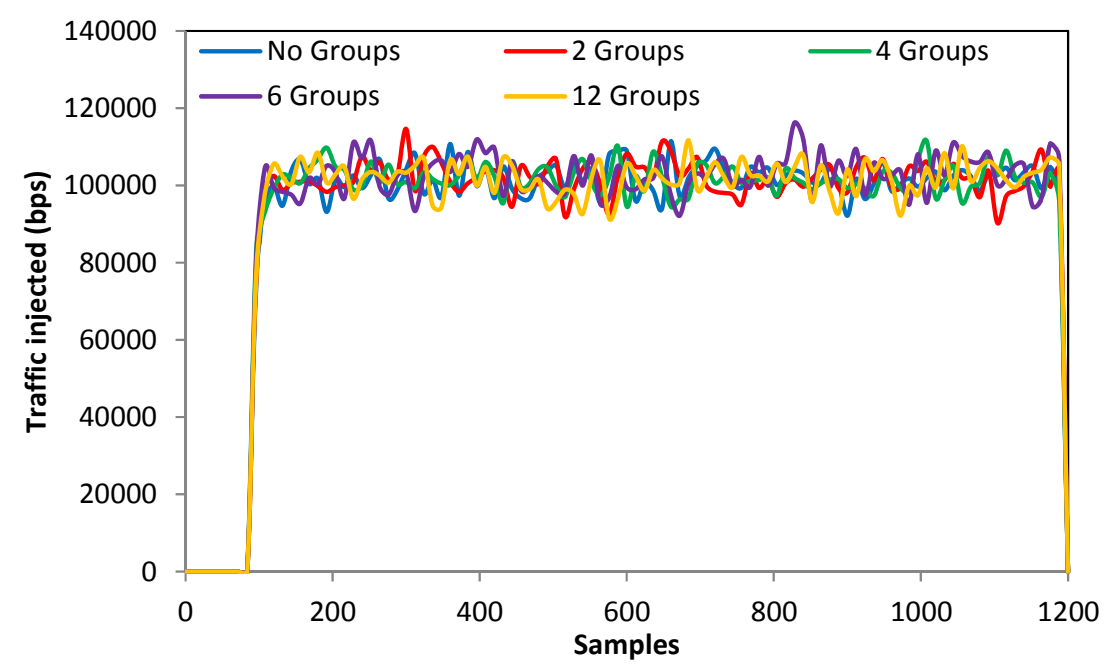

Figure 6. Injected traffic comparison.

Figure 7 and 8 show the total traffic in the WSN. In figure 7, the total traffic in the network is too high compared with the topology that uses collaborative groups. When the network does not have collaborative groups, the average total traffic is around $6500 \mathrm{Kbps}$, when we have 2 cooperative groups it decreases 95\%. In figure 8 , we only see the cooperative group-based topologies in order to show better the results. The total traffic decreases when the number of groups increases. As we can see in Figure 8, when we have 2 groups the average total traffic is around $310 \mathrm{Kbps}$, but when we have more groups (e.g. 6 groups), the total traffic decreases down to $140 \mathrm{Kbps}$. This demonstrates that collaborative group-based WSNs have lower traffic. 


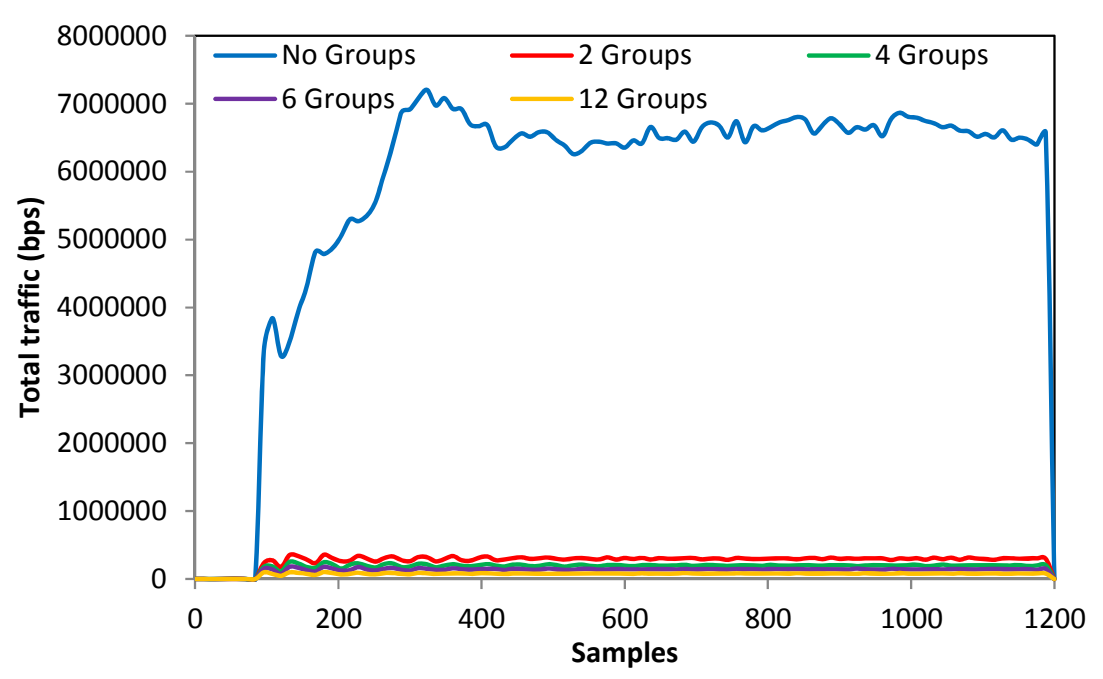

Figure 7. Total traffic comparison.

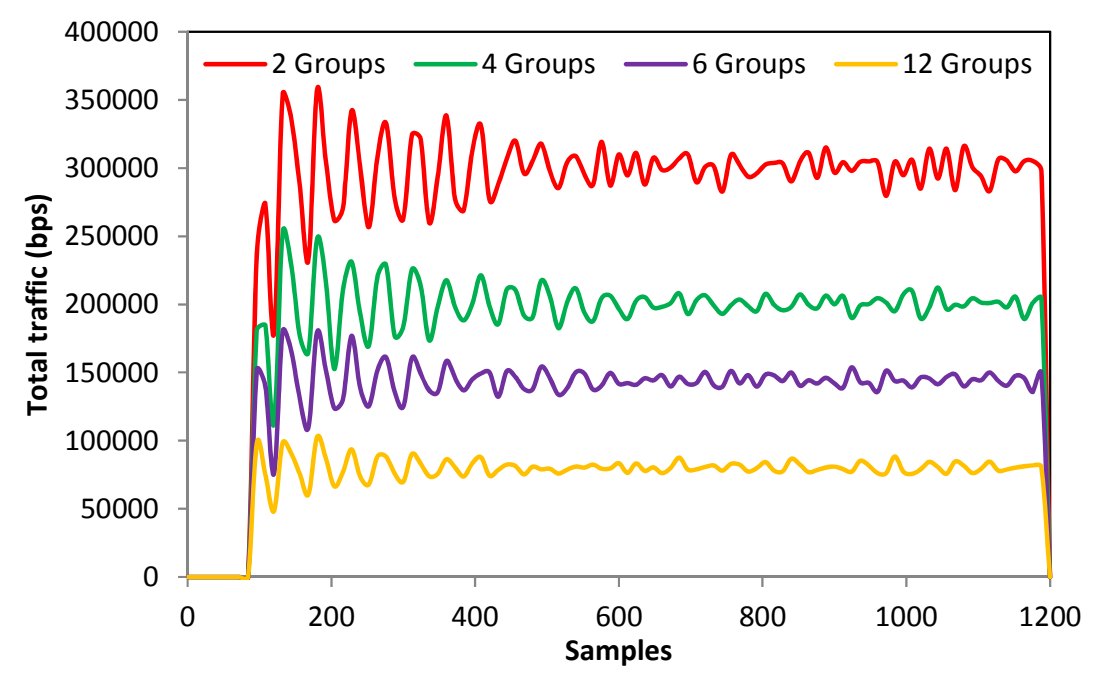

Figure 8. Total traffic comparison of cooperative group-based WSNs.

Figure 9 and 10 show the delay in the network. In Figure 9, we can see that the difference between cooperative group-based WSNs and a regular WSN is very high. In a regular WSN, the delay is around 120 seconds (which is too high for any application). This high value is obtained because the mobility of nodes. Constant mobility causes that the nodes need to compute their routing table constantly, and this process takes more time to arrive at the convergence state. This delay could change according to the used routing protocol. Anyway, its difference with cooperative group-based WSN is high.

In Figure 10 we can see the delay when we apply cooperative groups in the WSN. In all cases the delay is lower than 0.05 seconds. There is a peak at around 100 samples, because in this moment the simulation starts. Then, when the WSN converges the delay is lower and very constant. There is quite difference between 
cooperative group-based WSN and regular WSN. When there are not collaborative groups, the nodes are not segmented, and, for this reason, we need more resources to manage the network. When the WSN is divided into cooperative groups, the management process is also divided, for this reason we need less resources.

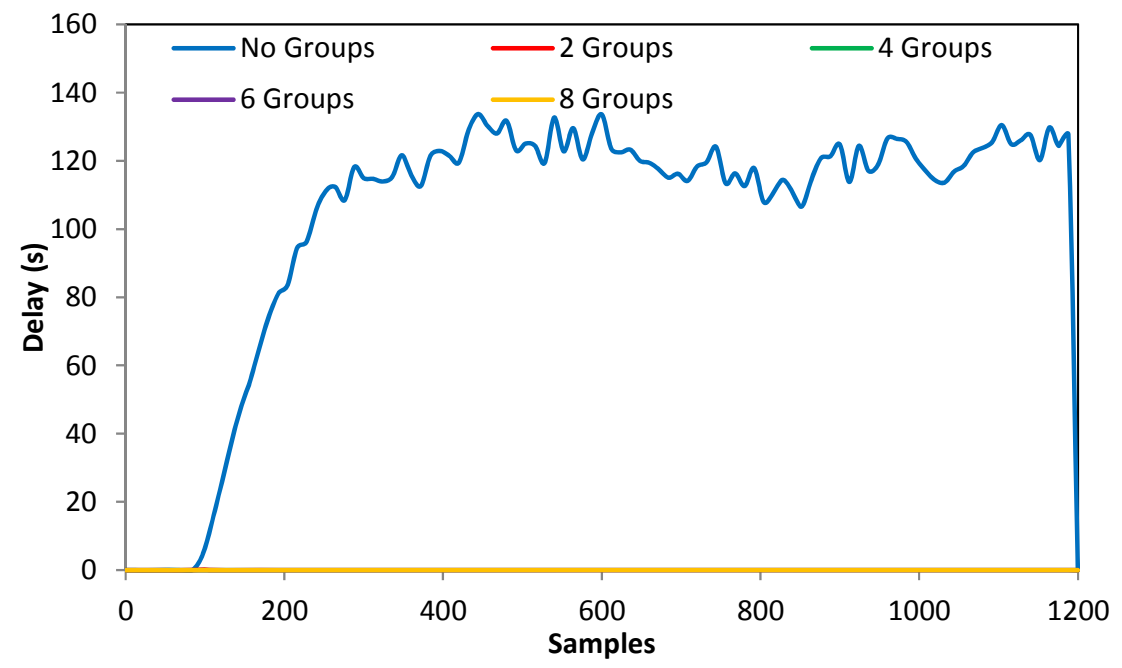

Figure 9. Delay comparison.

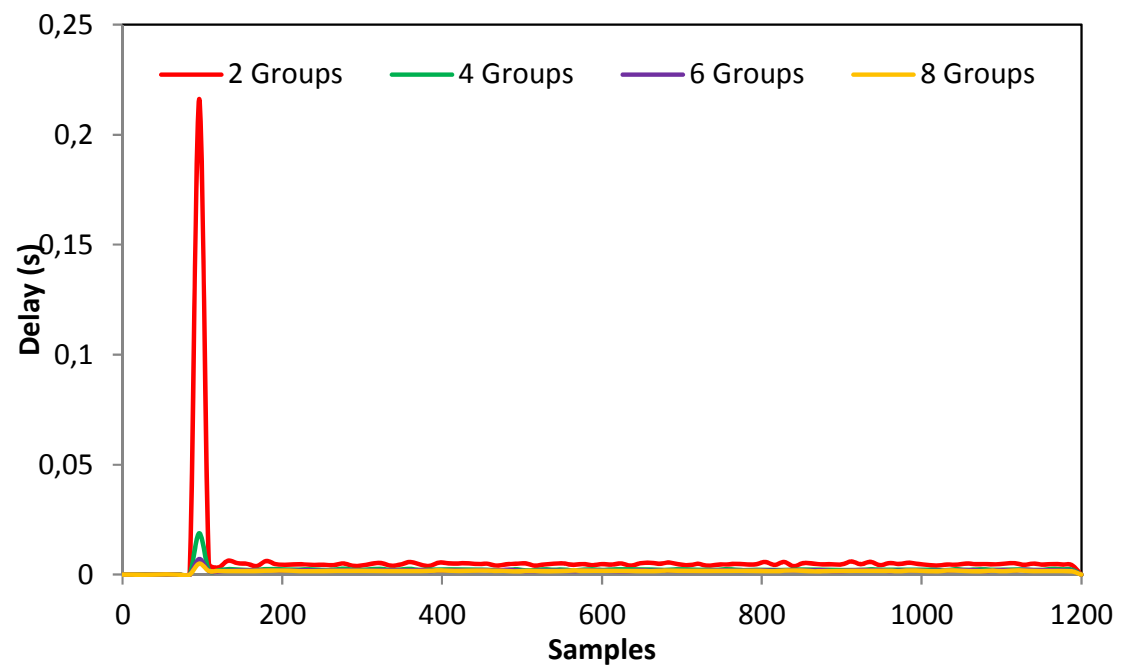

Figure 10. Delay comparison of cooperative group-based WSNs.

In Figure 11, we show the average number of hops needed to arrive to a destination when we are using groups and when we are not. As we can see, when we have a regular WSN, the average number of hops is around 6, so we need to cross 6 nodes to arrive to the destination. When we have collaborative groups in the network, the average number of hops is the half. We need 3 hops to arrive to the destination when we use collaborative group-based topologies. 


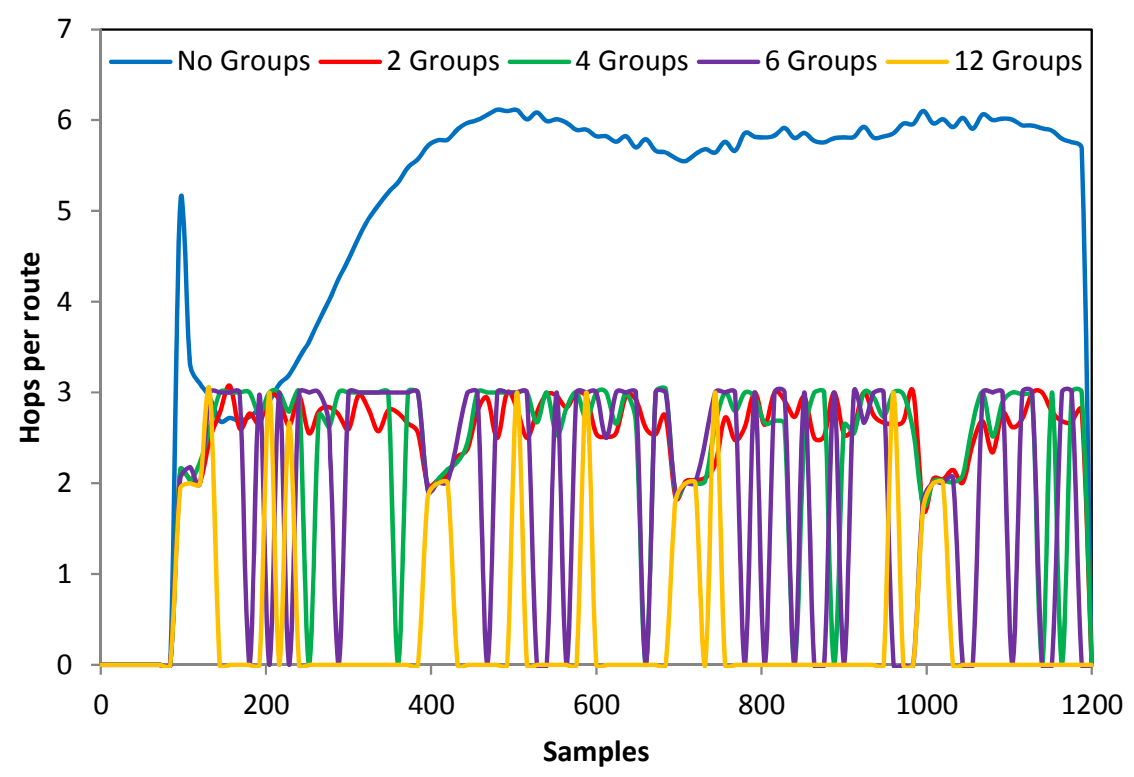

Figure 11. Hops per route needed to arrive to the destination.

In figure 12 and 13 we analyze the number of ACKs sent in regular and groupbased WSNs. In these cases we observe the same behavior as in previous figures. The regular architecture needs more ACKs (2400) than the cooperative groupbased architectures (see Figure 12). This is because regular WSNs need more messages to manage the architecture.

In order to better see the total number of ACKs sent in cooperative group-based WSNs, we show figure 13. In this figure we can see that the number of ACKs sent follow the same pattern for all topologies independently of the number of groups, although each topology inserts more or less ACKs. When we have 2 groups, where each group manages 50 sensor nodes, the average total ACKs sent is around 400. This number drops to half when the number of groups is equal to 4 . But, figure 13 shows that although we increase the number of groups, the total ACKs sent will not be less than a certain value. In this case, for a topology with 6 or 12 groups, the total number of ACK sent approximately equals 75 . 


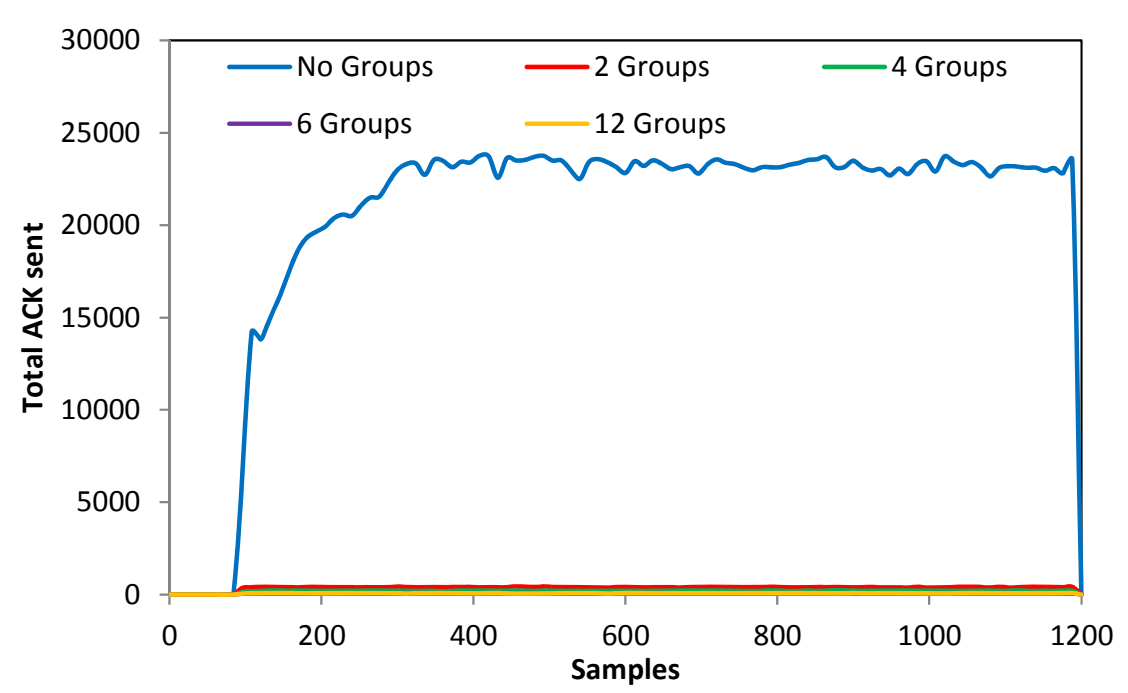

Figure 12. Total number of ACKs sent.

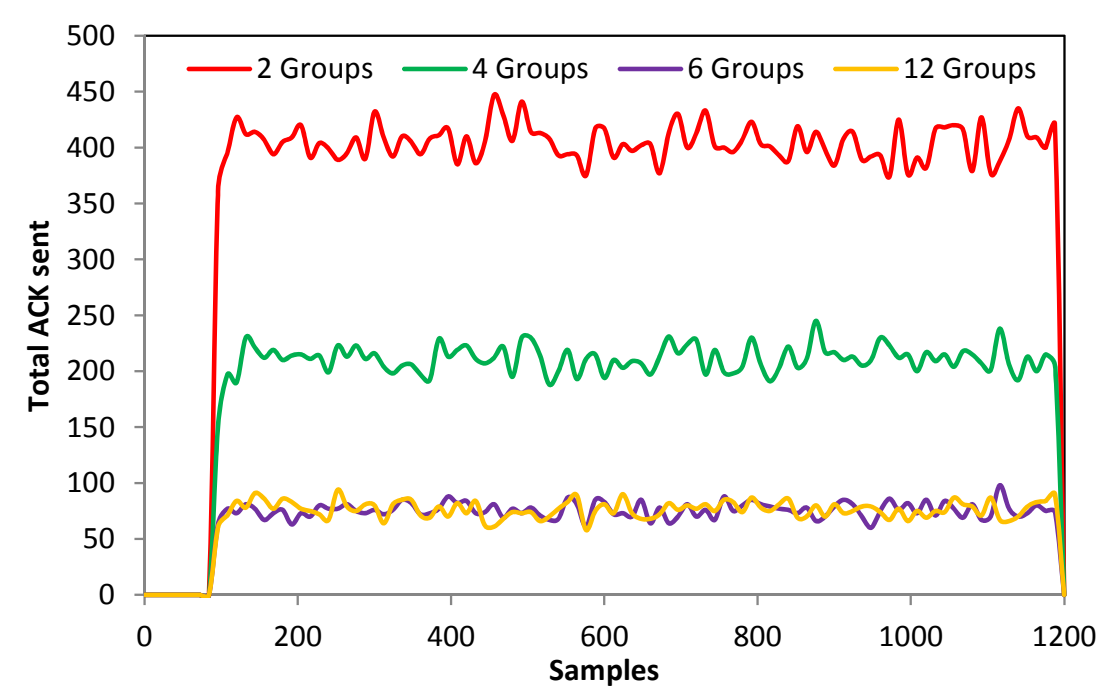

Figure 13. Total number of ACKs sent in cooperative group-based WSNs.

Figures 14 and 15 show the retransmission attempts for all cases aforementioned. In figure 14 we see that the regular WSN needs approximately 1.5 retransmission packets to guarantee the correct running of the system. But this retransmission is not needed when our system is based on collaborative groups, because the required management is done by cooperative group-based WSN.

When we increase the number of groups we need less number of retransmissions. When we have two groups, the average number of retransmissions is less than 0.1 packets, so it is negligible (see Figure 15). We can notice that when the number of groups is less than 4 , the retransmission packets could be zero. Observing these simulations (Figure 14 and 15) we can affirm that when we use cooperative 
groups in our WSNs, we needs less retransmissions, even they could be zero, depending of the system.

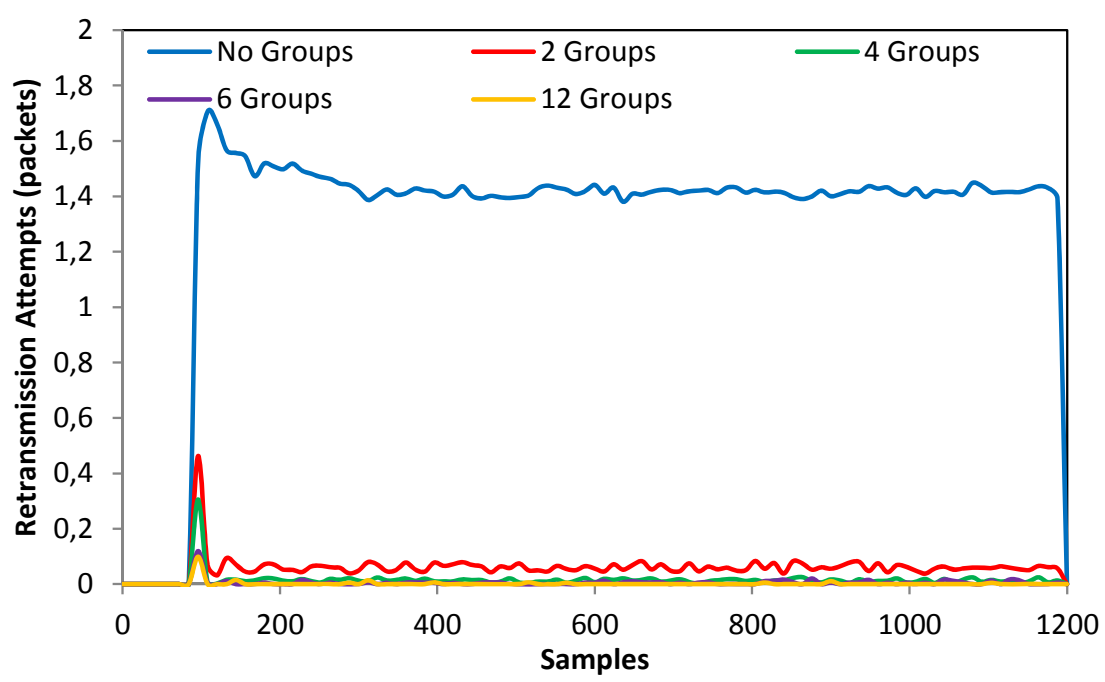

Figure 14. Retransmission Attempts.

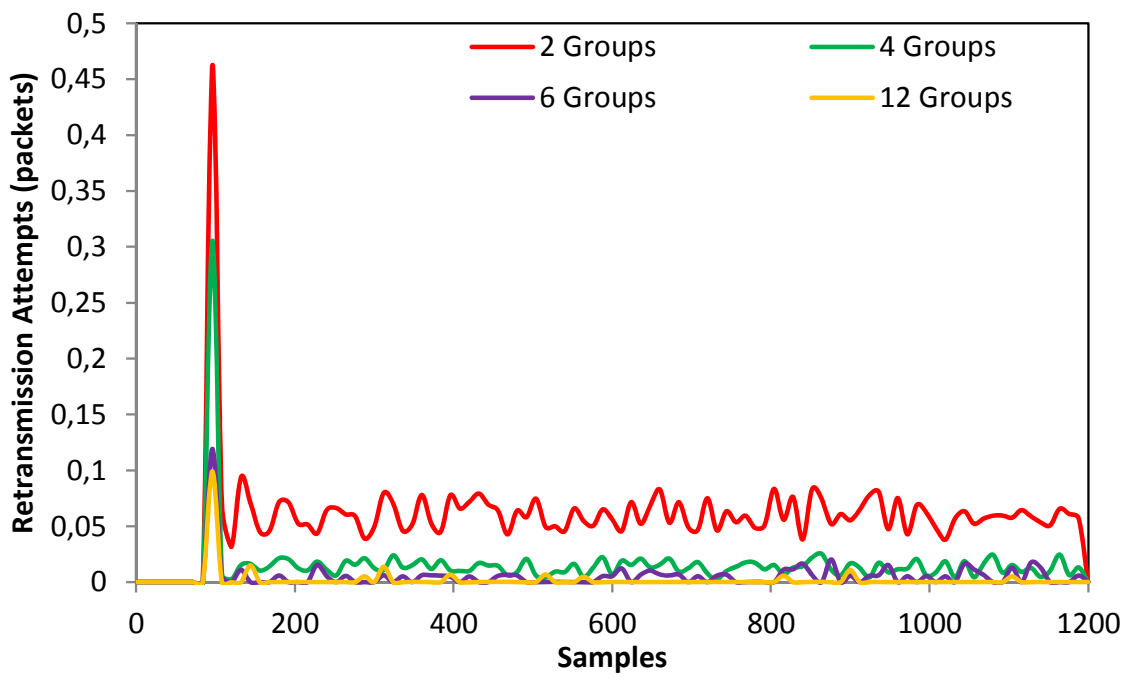

Figure 15. Retransmission Attempts in cooperative group-based WSNs.

Finally, in Figure 16, we present the load processed by a collaborative group. In this figure we only focus on the group-based WSNs, because, as we have seen in the previous figures, the regular WSN has worse performance. In this figure we see that when we have 2 groups in our network, the load is around $150 \mathrm{Kbps}$, this load decreases down to $60 \mathrm{Kbps}$ when we have 4 groups, $25 \mathrm{Kbps}$ for 6 groups, and less than $10 \mathrm{kbps}$ for 12 groups. This happens because when we have more collaborative groups, the number of nodes managed per group is lower. When we have a lot of collaborative groups in the WSN, we need more control information to manage it correctly. For this reason, when we select the number of groups, we 
should think several issues: to take into account the efficiency at network level, and take care of the management information needed to create and manage each collaborative group.

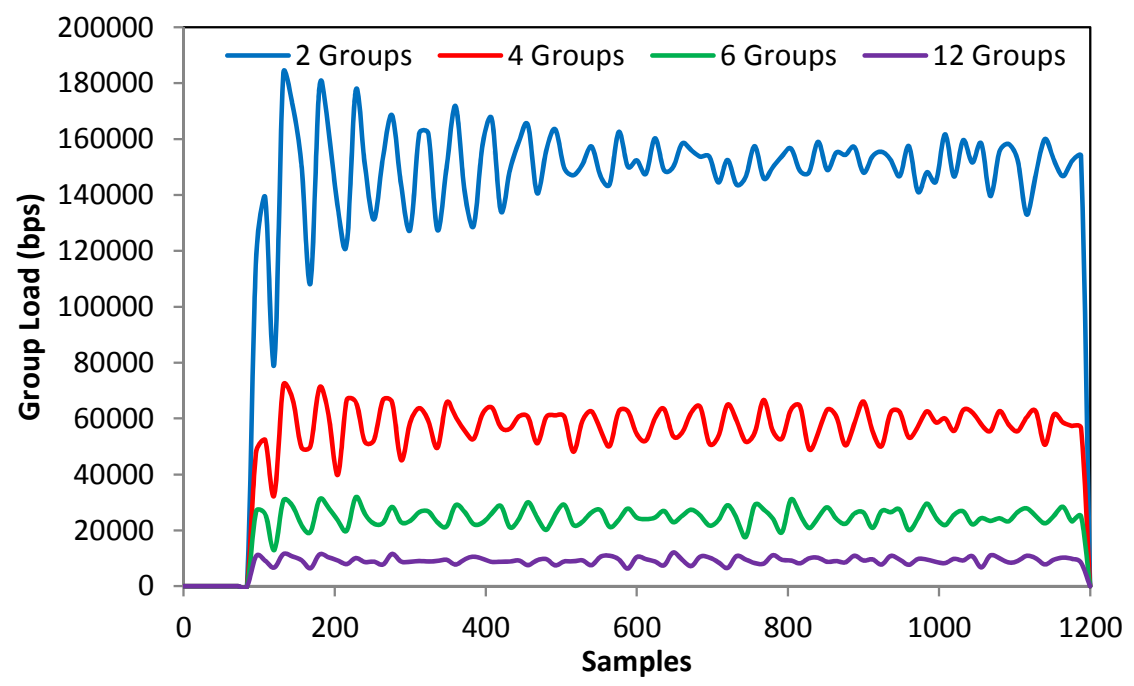

Figure 16. Load processed by a group.

\section{Conclusion and Future Work}

In this paper we have analyzed cooperative group-based WSNs. In this type of WSNs when a sensor detects a new event, the alert is sent to its group and it is distributed to an appropriate neighboring groups based on the information shared between sensors. Cooperation between groups could be used to change the direction of the alert propagation and the level of the alert in order to take the appropriate actions.

Using several analytical analyses we have proved that the cooperative groupbased WSNs save energy and improve the efficiency of the WSN communications. Moreover, we have seen that there are some WSN topologies that have better results than others. For this reason, our future work is based on this issue. We will analyze the best collaborative group-based WSN topology. Moreover, in future works we will study the energy issues related with mobile sensors and the communication procedures of joining and leaving the groups.

\section{References}

[1] Akyildiz, I.F., Su, W., Sankarasubramaniam, Y., Cayirci, E. (2002). Wireless sensor networks: a survey Journal of Computer Networks, 38 (4) 393-422. 
[2] Miguel Garcia, Diana Bri, Sandra Sendra, Jaime Lloret, Practical Deployments of Wireless Sensor Networks: a Survey, Journal On Advances in Networks and Services. Vol. 3 Issue 1\&2. Pp. 1-16. 2010.

[3] Lloret J., Garcia M., Bri D., Sendra S. (2009) A Wireless Sensor Network Deployment for Rural and Forest Fire Detection and Verification. Sensors. 911 8722-8747.

[4] Mainwaring, A., Polastre, J., Szewczyk, R., Culler, D. (2002). Wireless sensor networks for habitat monitoring. In ACM Workshop on Sensor Networks and Applications (WSNA'02), Atlanta, GA, USA, September.

[5] Miguel Garcia, Sandra Sendra, Gines Lloret, Jaime Lloret, Monitoring and Control Sensor System for Fish Feeding in Marine Fish Farms. IET Communications. 2010. In press.

[6] Sinha, A., Chandrakasan, A. (2001). Dynamic power management in wireless sensor networks. IEEE Design and Test of Computers, 18 (2) 62-74.

[7] Garcia, M., Coll, H., Bri, D., Lloret, J. (2008). Using MANET protocols in Wireless Sensor and Actor Networks. The Second International Conference on Sensor Technologies and Applications (SENSORCOMM 2008). Cap Esterel, Costa Azul (Francia). 25-31 August.

[8] Lloret, J., García, M., Boronat, F., Tomás, J. (2008). Chapter 13: MANET protocols performance in group-based Networks. Wireless and Mobile Networking. Springer Berlin Heidelberg Boston. Vol.284, Pp. 161-172.

[9] Lloret, J., García, M., Tomás, J. (2008). Improving Mobile and Ad-Hoc Networks Performance using Group-based topologies. Wireless Sensor and Actor Networks 2008 (WSAN 2008). Springer Berlin Heidelberg New York. Ottawa (Canada). 14-15 July.

[10] Lloret, J., Palau, C., Boronat, F., Tomas, J. (2008). Improving Networks Using Group-based Topologies. Journal of Computer Communications. Elsevier B. V. 31 (14) 3438-3450 .

[11] Lloret, J., Garcia, M., Tomás,J., Boronat, F. (2008). GBP-WAHSN: A Group-Based Protocol for Large Wireless Ad Hoc and Sensor Networks. Journal of Computer Science and Technology. 23 (3) 461-480.

[12] Lloret, J., García, M., Boronat, F., Tomás, J. (2008). MANET protocols performance in group-based Networks. 10th IFIP International Conference on Mobile and Wireless Communications Networks (MWCN 2008). Toulouse (France). 30 September -2 October.

[13] Garcia, M., Sendra, S., Lloret, J., Lacuesta, R. (2010) Saving energy with cooperative groupbased wireless sensor networks. Cooperative Design, Visualization, and Engineering: CDVE 2010. LNCS. Vol. 6240. Pp. 231-238. September 2010.

[14] Lloret, J., Sendra, S., Coll, H., García, M. (2010). Saving Energy in Wireless Local Area Sensor Networks. The Computer Journal (2010) 53(10): 1658-1673. Oxford University Press.

[15] Meiyappan, S. S., Frederiks, G., Hahn, S. (2006). Dynamic Power Save Techniques for Next Generation WLAN Systems. Proceedings of the 38th Southeastern Symposium on System Theory (SSST), Cookeville, Tennessee, USA, 5-7 March.

[16] Raghunathan, V., Schurgers, C., Park, S., Srivastava, M. (2002). Energy aware wireless microsensor networks. IEEE Signal Processing Magazine, 19 (2) 40-50. 
[17] Rex Min, Manish Bhardwaj, Seong-Hwan Cho, Eugene Shih, Amit Sinha, Alice Wang, Anantha Chandrakasan, (2001). Low power wireless sensor networks. Proceedings of International Conference on VLSI Design, Bangalore, India, 3-7 January.

[18] Salhieh, A., Weinmann, J., Kochha, M., Schwiebert,L. (2001). Power efficient topologies for wireless sensor networks. Procedimgs of the IEEE International Conference on Parallel Processing. Pp 156-163. 3-7 September

[19] Jayashree, S., Manoj, B. S., Murthy, C.S. R. (2004) A battery aware medium access control (BAMAC) protocol for Ad-hoc wireless network, Proceedings of the 15th IEEE International Symposium on Personal, Indoor and Mobile Radio Communications (PIMRC 2004). Barcelona (Spain), 5-8 September, Vol. 2, Pp. 995-999.

[20] Ye, W., Heidemann, J., Estrin. D. (2002) An energy-efficient MAC protocol for wireless sensor networks. Proceedings IEEE INFOCOM 2002, The 21st Annual Joint Conference of the IEEE Computer and Communications Societies, New York, USA, 23-27June.

[21] Ching, C., Schindelhauer, C. (2010) Utilizing detours for energy conservation in mobile wireless networks. Journal of Telecommunication Systems. Springer Netherlands. (DOI 10.1007/s11235-009-9188-3)

[22] Gao, Q., Blow, K., Holding, D., Marshall, I., Peng, X. (2004) Radio range adjustment for energy efficient wireless sensor networks, Journal of Ad Hoc Networks. 4 (1) 75-82.

[23] Deying Li, Xiaohua Jia, and Hai Liu. (2004). Energy Efficient Broadcast Routing in Static Ad Hoc Wireless Networks. IEEE Transactions on Mobile Computing, Vol. 3, No. 1, pp. 1-8. January-March 2004.

[24] Camilo, Tiago and Carreto, Carlos and Silva, Jorge and Boavida, Fernando. (2006). An Energy-Efficient Ant-Based Routing Algorithm for Wireless Sensor Networks. Lecture Notes in Computer Science, Ant Colony Optimization and Swarm Intelligence, vol. 4150, pp. 49-59.

[25] M. Younis, M. Youssef, and K. Arisha. (2002). Energy-Aware Routing in Cluster-Based Sensor Networks. In Proceedings of the 10th IEEE International Symposium on Modeling, Analysis, and Simulation of Computer and Telecommunications Systems (MASCOTS '02). IEEE Computer Society, Washington, DC, USA, pp. 129-136.

[26] Zhao Cheng, Mark Perillo, and Wendi B. Heinzelman. (2008). General Network Lifetime and Cost Models for Evaluating Sensor Network Deployment Strategies. IEEE TRANSACTIONS ON MOBILE COMPUTING, VOL. 7, NO. 4, pp. 484-497. April 2008.

[27] Nojeong Heo and Pramod K. Varshney. (2005).Energy-Efficient Deployment of Intelligent Mobile Sensor Networks. IEEE Transactions on Systems, Man, and Cybernetics—Part A: Systems And Humans, Vol. 35, No. 1, pp. 78-92. January 2005.

[28] Vlajic, N., Xia, D. (2006) Wireless sensor networks: to cluster or not to cluster?. International Symposium on a World of Wireless, Mobile and Multimedia Networks, 2006. WoWMoM 2006.

[29] Garcia, M. and Lloret, J. (2009) A Cooperative Group-Based Sensor Network for Environmental Monitoring. Cooperative Design, Visualization, and Engineering: CDVE 2009. LNCS 5738, pp. 276-279. 
[30] Garcia, M., Bri, D., Boronat, F., Lloret, J. (2008) A New Neighbour Selection Strategy for Group-Based Wireless Sensor Networks. In: 4th Int. Conf. on Networking and Services, ICNS 2008, March 16-21, pp. 109-114

[31] Kaplan, E.D. (1996) Understanding GPS: Principles and Applications. Artech House, Boston. [32] Stojmenovic, I. (2002) Position based routing in ad hoc networks. IEEE Communications Magazine 40(7), 128-134.

[33] Heinzelman, W.B., Chandrakasan, A.P., Balakrishnan H. (2002) An application-specific protocol architecture for wireless microsensor networks, IEEE Trans. on Wireless Communications 1 (4) 660-670.

[34] Bhardwaj, M., Garnett, T., Chandrakasan, A.P. (2001) Upper bounds on the lifetime of sensor networks. International Conference on Communications (ICC'01), June 2001, pp. 785-790.

[35] A. Gibbons. (1985) Algorithmic graph theory. Cambridge University Press, 1985.

[36] Fraigniaud, P., Pelc, A., Peleg, D., Perennes, S. (2000) Assigning labels in unknown anonymous networks. Proceedings of the 19th Annual ACM SIGACT-SIGOPS Symposium on Principles of Distributed Computing, Portland, OR, USA, July 2000, vol. 1, pp. 101-111.

[37] OPNET Modeler ${ }^{\circledR}$ Wireless Suite network simulator, available at http://www.opnet.com/solutions/network_rd/modeler_wireless.html 